\title{
WEYL MATRIX FUNCTIONS AND INVERSE PROBLEMS FOR DISCRETE DIRAC-TYPE SELF-ADJOINT SYSTEMS: EXPLICIT AND GENERAL SOLUTIONS
}

\author{
B. Fritzsche, B. Kirstein, I. RoItBerg, A. L. SAKhNOVICH
}

\begin{abstract}
It is shown that the discrete Dirac-type self-adjoint system is equivalent to the block Szegö recurrence. A representation of the fundamental solution is obtained, inverse problems on the interval and semiaxis are solved. A Borg-Marchenko type result is obtained, too. Connections with block Toeplitz matrices are treated
\end{abstract}

Mathematics subject classification (2000): 39A12, 37K35, 47B35.

Key words and phrases: Discrete Dirac system, Szegö recurrence, Weyl function, inverse problem, $j$-theory, block Toeplitz matrix.

\section{REFERENCES}

[1] D. ALPAY, I. GOHBERG, Inverse spectral problems for difference operators with rational scattering matrix function, Integral Equations Operator Theory 20 (1994), 125-170.

[2] D. ALPAY, I. GOHBERG, Inverse spectral problem for differential operators with rational scattering matrix functions, J. Differ. Equations 118 (1995), 1-19.

[3] D. Alpay, I. Gohberg, Connections between the Carathéodory-Toeplitz and the Nehari extension problems: the discrete scalar case, Integral Equations Operator Theory 37 (2000), 125-142.

[4] D. Alpay, I. GohBerg, Inverse problems associated to a canonical differential system, in: Oper. Theory, Adv. Appl. 127 (2001), Birkhäuser, 1-27.

[5] D. AlPAY, I. GOHBERG, Discrete analogs of canonical systems with pseudo-exponential potential. Definitions and formulas for the spectral matrix functions, in: Oper. Theory, Adv. Appl. 161 (2005), Birkhäuser, 1-47.

[6] D. AlPAY, I. Gohberg, Discrete analogs of canonical systems with pseudo-exponential potential. Inverse Problem, in: Oper. Theory, Adv. Appl. 165 (2005), Birkhäuser, 31-65.

[7] D. Alpay, I. Gohberg, Discrete systems and their characteristic spectral functions, Mediterr. J. Math., 4 (2007), 1-32.

[8] D. AlPay, I. Gohberg, M.A. Kaashoek, A.L. Sakhnovich, Direct and inverse scattering problem for canonical systems with a strictly pseudo-exponential potential, Math. Nachrichten 215 (2000), $5-31$.

[9] T. AKTOSun, M. Klaus, C. VAN DER MEE, Direct and inverse scattering for selfadjoint Hamiltonian systems on the line, Integral Equations Operator Theory 38 (2000) 129-171.

[10] D.Z. ARov, H. DyM, J-inner matrix functions, interpolation and inverse problems for canonical systems V, Integral Equations Operator Theory 43 (2002) 68-129.

[11] H. BART, I. GOHBERG, M.A. KAASHOEK, Minimal factorization of matrix and operator functions, Operator Theory: Adv. and Appl. 1, Birkhäuser Verlag, Basel, 1979.

[12] S. ClarK, F. GeSZTESY, W. RENGER, Trace formulas and Borg-type theorems for matrix-valued Jacobi and Dirac finite difference operators, J. Differ. Equations 219 (2005), 144-182.

[13] S. Clark, F. GeSZTESY, M. ZinChenko, Weyl-Titchmarsh theory and Borg-Marchenko-type uniqueness results for CMV operators with matrix-valued Verblunsky coefficients, Operators and Matrices 1 (2007), 535-592. 
[14] M.J. CoRless, A.E. Frazho, Linear Systems and Control - An Operator Perspective, Marcel Dekker, New York, 2003.

[15] Ph. Delsarte, Y. Genin, Y. Kamp, Orthogonal polynomial matrices on the unit circles, IEEE Trans. Circuits and Systems, CAS-25 (1978), 149-160.

[16] Ph. Delsarte, Y. Genin, Y. Kamp, Schur parametrization of positive definite block-Toeplitz systems, SIAM J. Appl. Math. 36:1 (1979), February, 34-46.

[17] V.K. Dubovoj, B. Fritzsche, B. Kirstein, Matricial version of the classical Schur problem, in: Teubner-Texte zur Mathematik [Teubner Texts in Mathematics]129, B. G. Teubner Verlagsgesellschaft mbH, Stuttgart, 1992.

[18] H. DYM, Hermitian block Toeplitz matrices, orthogonal polynomials, reproducing kernel Pontryagin spaces, interpolation and extension, in: Oper. Theory, Adv. Appl. 34 (1988), Birkhäuser, 79-135.

[19] H.DYM, J -contractive matrix functions, reproducing kernel Hilbert spaces and interpolation, CBMS Regional Conference Series in Mathematics 71, American Mathematical Society, Providence, RI, 1989.

[20] B. Fritzsche, B. KIRSTEIN, An extension problem for non-negative Hermitian block Toeplitz matrices, Math. Nachr., Part I: 130 (1987), 121-135; Part II: 131 (1987), 287-297; Part III: 135 (1988), 319-341; Part IV: 143 (1989), 329-354; Part V: 144 (1989), 283-308.

[21] B. FRITZSCHE, B. KIRSTEIN, On the Weyl matrix balls associated with nondegenerate matrix-valued Carathéodory functions, Z. Anal. Anwendungen 12 (1993), 239-261.

[22] B. Fritzsche, B. Kirstein, M. Mosch, On block completion problems for Arov-normalized $j_{q q}-J_{q}$-elementary factors, Linear Algebra Appl. 346 (2002), 273-291.

[23] B. FRITZSCHE, B. KIRSTEIN, K. MÜLLER, An analysis of the block structure of certain subclasses of $j_{q q}$-inner functions, Z. Anal. Anwendungen 17 (1998), 459-478.

[24] B. FritzSCHE, B. KirSTEIN, A.L. SAKHNOVICH, Completion problems and scattering problems for Dirac type differential equations with singularities, J. Math. Anal. Appl. 317 (2006), 510-525.

[25] J.S. GERONIMO, Polynomials orthogonal on the unit circle with random recurrence coefficients, in: Lecture Notes in Math. 1550 (1993), Springer, Berlin, 43-61.

[26] J.S. Geronimo, F. GESZTESY, H. HOLDEn, Algebro-geometric solutions of the Baxter-Szegö difference equation, Comm. Math. Phys. 258 (2005), 149-177.

[27] F. Gesztesy, H. Holden, J. Michor, G. TesChl, Algebro-Geometric Finite-Gap Solutions of the Ablowitz-Ladik Hierarchy, arxiv: nlin.SI/0611055 (Int. Math. Res. Not. to appear).

[28] F. Gesztesy, B. Simon, On local Borg-Marchenko uniqueness results, Commun. Math. Phys. 211 (2000), 273-287.

[29] I.GohBERG, M.A.KAASHOEK, A.L.SAKHNOVICH, Canonical systems with rational spectral densities: explicit formulas and applications, Math. Nachrichten 194 (1998), 93-125.

[30] I. GoHBERG, M.A. KAASHOEK, A.L. SAKHNOVICH, Scattering problems for a canonical system with a pseudo-exponential potential, Asymptotic Analysis 29:1 (2002), 1-38.

[31] L. GolinsKII, P. NeVAI, Szegö difference equations, transfer matrices and orthogonal polynomials on the unit circle, Comm. Math. Phys. 223 (2001), 223-259.

[32] M.A. KAASHOEK, A.L. SAKHNOVICH, Discrete skew self-adjoint canonical system and the isotropic Heisenberg magnet model, J. Functional Anal. 228 (2005), 207-233.

[33] B.M. LEVITAN, I.S. SARGSJAN, Introduction to spectral theory: selfadjoint ordinary differential operators, Translations of Mathematical Monographs, Vol. 39, American Mathematical Society, Providence, R.I., 1975.

[34] V.P. POTAPOV, The multiplicative structure of $J$-contractive matrix functions Am. Math. Soc. Transl. II Ser. 15 (1960), 131-243.

[35] J. RovNyAK, L.A. SAKHNOvich, Some indefinite cases of spectral problems for canonical systems of difference equations, Linear Algebra Appl. 343/344 (2002), 267-289.

[36] A.L. SAKHNovich, On the continuation of the block Toeplitz matrices, Functional Analysis (Uljanovsk) 14 (1980), 116-127.

[37] A.L. SAKHNOVICH, Exact solutions of nonlinear equations and the method of operator identities, Linear Algebra Appl. 182 (1993), 109-126.

[38] A.L. SAKHNovich, Dressing procedure for solutions of nonlinear equations and the method of operator identities, Inverse Problems 10 (1994), 699-710.

[39] A.L. SAKHNOVICH, Iterated Bäcklund-Darboux transform for canonical systems, J. Functional Anal. 144 (1997), 359-370.

[40] A.L. SAKHNOVICH, Toeplitz matrices with an exponential growth of entries and the first Szegö limit theorem, J. Functional Anal. 171 (2000), 449-482.

[41] A.L. SAKHNOvich, Dirac type and canonical systems: spectral and Weyl-Titchmarsh fuctions, direct and inverse problems, Inverse Problems 18 (2002), 331-348. 
[42] A.L. SAKHNOVICH, Skew-self-adjoint discrete and continuous Dirac-type systems: inverse problems and Borg-Marchenko theorems, Inverse Problems 22 (2006), 2083-2101.

[43] L.A. SAKHNOVICH, On the factorization of the transfer matrix function, Dokl. Akad. Nauk SSSR 226 (1976), 781 - 784 . English transl. in Sov. Math. Dokl. 17 (1976), 203-207.

[44] L.A. SAKHNOVICH, Interpolation theory and its applications, Mathematics and its Applications 428 , Kluwer Academic Publishers, Dordrecht, 1997.

[45] L.A. SAKHNOVICH, Spectral theory of canonical differential systems, method of operator identities, Oper. Theory, Adv. Appl. 107, Birkhäuser Verlag, Basel-Boston, 1999.

[46] B. SIMON, A new approach to inverse spectral theory I. Fundamental formalism, Ann. of Math. 150 (1999), 1029-1057.

[47] B. SIMON, Orthogonal polynomials on the unit circle, Parts 1,2, Colloquium Publications, American Mathematical Society 51, 54, Providence, RI, 2005.

[48] G. TESCHL, Jacobi operators and completely integrable nonlinear lattices, Mathematical Surveys and Monographs 72, Providence, RI: AMS, 2000. 